\title{
Femur Bone Segmentation using a Pressure Analogy
}

\author{
Thamer S. Alathari, Mark S. Nixon \\ School of Electronics and Computer Science \\ University of Southampton, UK \\ [tsa1g11|msn]@soton.ac.uk
}

\author{
Mamadou T. Bah \\ Faculty of Engineering and the Environment \\ University of Southampton, UK \\ msn@soton.ac.uk
}

\begin{abstract}
It has been recently shown that preclinical analysis of computed tomography 3D image volumes can provide essential information to find the optimal position of an implant in hip replacement procedures. In order to extract such data, proper segmentation is crucial. Many of the currently-available methods depend on manually segmented data as the first step. Inherent difficulties concern the similar density of adjacent structures, and that physically-separated structures appear to touch in scanned imagery. In this study, we describe a new technique based on pressure analogy that depends on the local features of the image to accurately and automatically segment and visualize the femur bone and separate it from the acetabulum. The Dice coefficient was employed to study the similarity between the surface area of the segmentations compared with the manually segmented data, and a high value has been achieved. The same method also showed promising results in segmenting other limbs such as the pelvis, tibia and fibula bones.
\end{abstract}

Keywords-Visualization; Segmentation; 3D volume; Pressure; Analogy; Femur;

\section{INTRODUCTION}

Image segmentation is a valuable source of information for pre-clinical procedures often aiding hip replacement. Osteoarthritis, which is one of the most common types of arthritis, is a disease that causes the distance between the femur bone and the acetabulum (pelvis socket) to become very small due to the degradation of the articular cartilage and the overgrowth of the bone. For successful implant placement, a proper study for the positioning is essential to get the best results and to distribute the forces exerted by the body weight.

The problem of image segmentation in this scenario is illustrated in Fig. 1, which shows one slice of a stack of CTscanned images of the hip. The image shows the femoral head and the acetabulum, together with some surrounding tissue. We seek to process the whole volume image to visualize the femoral bone, and the image shows that the two bones appear to be connected.

Previous research has tackled this problem using manual segmentation as prerequisite such as; statistical shape methods to describe the femur bone [1] [2], general Hough transform to construct the optimum model [3] and iterative deformable

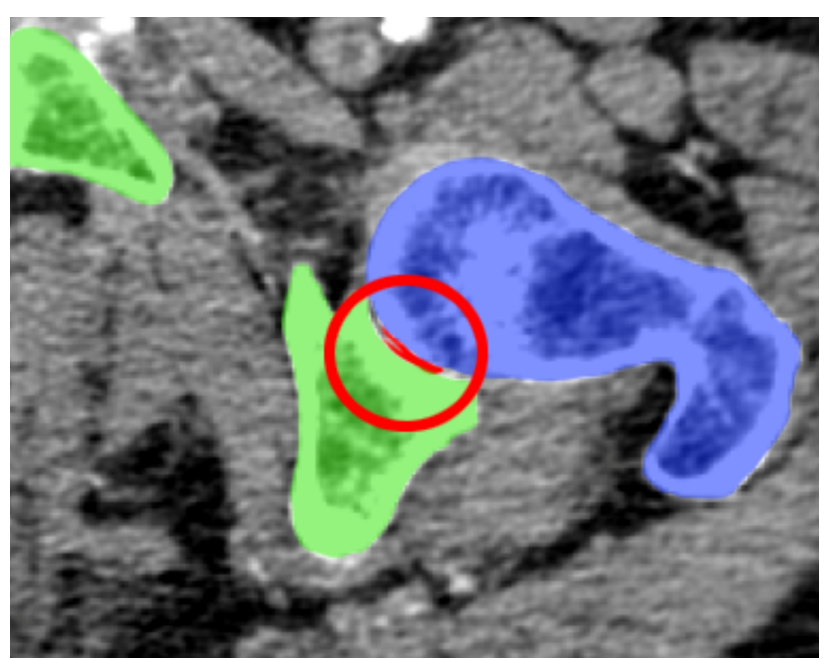

Fig. 1. The intersection between the femoral head (blue) with the acetabulum (green) is circled sand labeled in (red)

model to match the femur shape [4]. Other methods relied on initial parameters chosen by an operator to refine boundary information between the acetabulum and the femoral bone [5]. There are two automated approaches to bone segmentation in the literature, one is based on the graphcut method using statistical analysis and morphology [6] and the other one hinged on local adaptive thresholding conditioned on Bayesian rules [7].

In this work, we extend a new pressure analogy [8] to include segmentation of touching objects with similar density. Pressure is used as a volumetric analogy to provide an operator to analyze and refine features in volume data. Only the principles of pressure are used to motivate an image analysis operator: precise detail is not needed. Pressure domain data has been enhanced by a new mapping technique followed by morphological operations to expand the pressure mask effect. This is not possible using conventional morphological operations (without the pressure analogy) since these are isotropic and lack capability to separate gaps whilst retaining overall shape. Logical operations are applied to the pressure mask to separate the femur bone from the acetabulum. Labeling has been aided by using statistical operations. The proposed method is intuitive, anisotropic, noniterative and requires no previous knowledge and can 
automatically separate connected structures of similar appearance (or density, in CT images).

\section{FEATURE EXTRACTION USING THE PRESSURE ANALOGY}

Extracting the femur bone presents a great challenge for segmentation methods due to the existence of similar density bones surrounding and touching its surface. The pressure analogy presents a new perspective into the problem of separating connected objects in volumetric images. Unlike the previous work, which depends on the small gradient between objects, this paper addresses the separation of objects which have a similar density and which appear to be touching in that the space between them is often small and occasionally zero. The same basis as the original pressure approach [8] was adopted, one-sided threshold followed by a convolving cube to calculate the volume of the background to obtain pressure domain. The pressure domain provides valuable information about connected areas between the objects.

$$
\mathbf{P}_{x, y, z}=1 / \sum_{x, y, z \in \mathbf{W}} \mathbf{V}_{x, y, z} \quad \forall \mathbf{V}_{x, y, z} \in \text { background }
$$

where $\mathbf{W}$ is a window of size $5 \times 5 \times 5, \mathbf{V}$ is the volume thresholded so as to remove background data, retaining the high density values, and $\mathbf{P}$ is the pressure domain. The pressure data is then mapped using a high pass filter response to retain consistency and to provide a smooth transform. The filter is derived by arranging the pressure levels $p_{f}$ in ascending order and deriving a mapping function extracted from a high-pass filter:

$$
\boldsymbol{V}_{\boldsymbol{o}}=v_{i} \frac{Z_{R}}{Z_{R}+Z_{C}}
$$

where $\quad v_{i}=180, Z_{C}=-j\left(1 / 2 \pi p_{f} C\right), \quad Z_{R}=1500 \quad$ and $\mathrm{C}=1.06 \times 10^{-6}$ with a cut-off frequency $p_{f \text { cut off }}=100$, for $p_{f} \in[1,125]$, so the high pressure values in the top 25 levels are retained and emphasized. This mapping function is used to derive $\mathbf{P}^{\prime}=\mathbf{P}\left(\boldsymbol{V}_{\boldsymbol{o}}\right)$ which is then thresholded.

$$
\mathbf{M}_{x, y, z}=\mid \begin{array}{lr}
1 & \text { if } \mathbf{P}^{\prime}{ }_{x, y, z} \geq \text { threshold } \\
0 & \text { otherwise }
\end{array}
$$

After that, connectivity check was applied on the thresholded mask; each element labeled defines a structured element $\mathbf{M}_{i}$ stored in $4 \mathrm{D}$ matrix $\mathbf{M}_{\mathrm{L}}$. Each element is dilated three times to increase its mass and close the gap between elements using structure $\mathbf{S}$ of size $3 \times 3 \times 3$ with all voxels turned on.

$$
\mathbf{M}_{i}^{\prime}=\mathbf{M}_{i} \oplus \mathbf{S}
$$

\section{$\forall i \in 1$ : size $\mathbf{M}_{L}$ in the 4 th dimension}

The next step is to erode the whole mask in an attempt to minimize its effect.

$$
\begin{gathered}
\mathbf{M}_{\text {sum }}=\operatorname{logical}\left(\sum_{i=1}^{\# \text { elements }} \mathbf{M}_{i}^{\prime}\right) \\
\mathbf{M}_{\text {final }}=\mathbf{M}_{\text {sum }} \ominus \mathbf{S}
\end{gathered}
$$

The final mask $M_{\text {final }}$ is then inverted and applied to the thresholded volume $V$, separating the objects.

$$
\mathbf{S}_{x, y, z}=\neg \mathbf{M}_{\text {final }_{x, y, z}} \times \mathbf{V}_{\mathrm{x}, \mathrm{y}, \mathrm{z}}
$$

The objects were labeled individually to describe each object uniquely. The mask discards a small amount of data, which is implicit in these operations. To recover this data, the mask is multiplied by a logical thresholded volume to obtain only the areas in the mask (used to separate objects) and which do not exist in the background.

$$
\mathbf{R}_{x, y, z}=\mathbf{M}_{\text {final }_{x, y, z}} \times \operatorname{logical}\left(\mathbf{V}_{\mathrm{x}, \mathrm{y}, \mathrm{z}}\right)
$$

Skeletonization [9] was applied as per slice on the recovery volume $\mathbf{R}$ to create small creek between the mask segments to aid the reconstruction process. The labeled mask is then grown on those areas using statistical mode. A unique label now defines each object in volume $\mathbf{F}$, with no lost data.

$$
\mathbf{F}_{x, y, z}=\operatorname{Mode}\left(\mathbf{S}_{\boldsymbol{x}, \boldsymbol{y}, \mathbf{z} \in \boldsymbol{W}}\right) \quad \forall \boldsymbol{R}_{\boldsymbol{x}, \boldsymbol{y}, \boldsymbol{z}} \in \text { foreground }
$$

Three morphological closing operations have been applied to every labeled object and close any open gaps in the detected object.

$$
\mathbf{B}_{\text {final }, i}=\mathbf{F}_{i}^{\bullet} \mathbf{S} \quad \forall i \in 1 \text { : Number of labels }
$$

where $B_{\text {final }}$ is a $4 \mathrm{D}$ matrix contain the volume of each segmented object. To analyse the validity of the results, the surface area was calculated for the proposed method results $a_{p}$ and the manually segmented data $a_{m}$. The manual segmentation took place by thresholding then removing surrounding bones and soft tissue. The Dice similarity coefficient $(d c)$ [10] was used to compare the two results.

$$
d c=2 a_{p} /\left(a_{p}+\mathrm{a}_{m}\right)
$$

Our work describes a solution that is based on the original basis of the pressure analogy. Virtually the same advantages have been accrued as in the literature; a non-iterative, anisotropic method that depends on local features of the objects in the scene. The novel step is the high level refinement and the extension towards separating connected objects of similar density. 


\section{RESULTS}

As discussed previously, the method provides a mask that is used to separate the connected objects. In this section, we evaluate the performance of the proposed method on 51 volumetric cropped images of femur bone acquired using CT scanner. The images were cropped to concentrate the

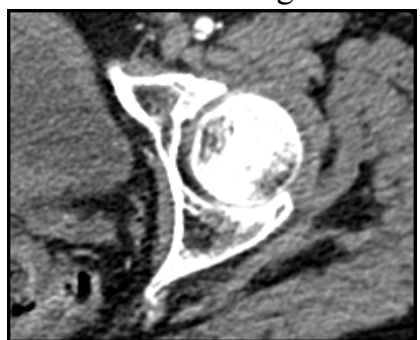

$\mathrm{z}=25$

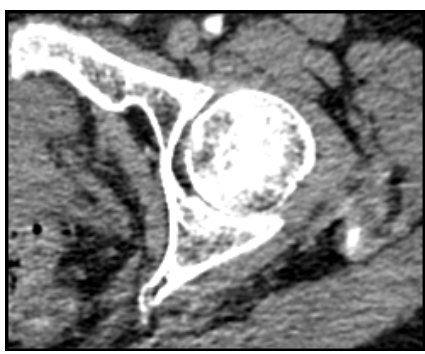

$\mathrm{z}=38$

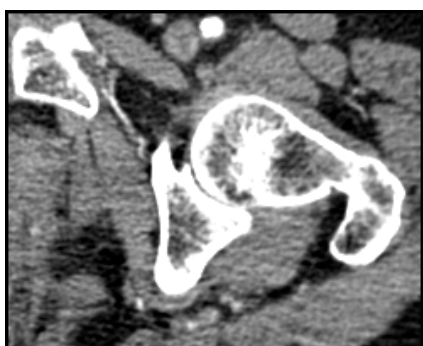

$\mathrm{z}=53$

Fig. 2. Different slice though the hip showing the contact area between the femoral head and the acetabulum

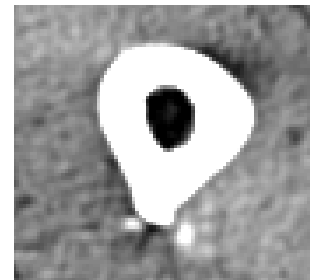

$z=325$

$\mathrm{z}=250$

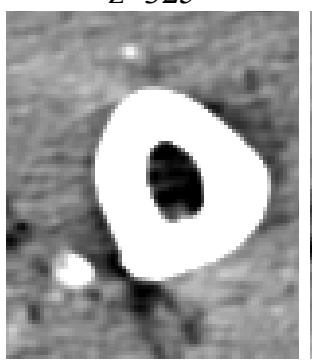

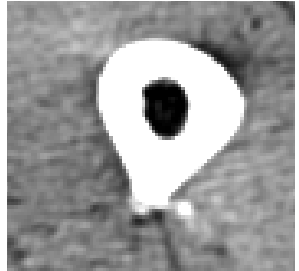

$\mathrm{z}=329$

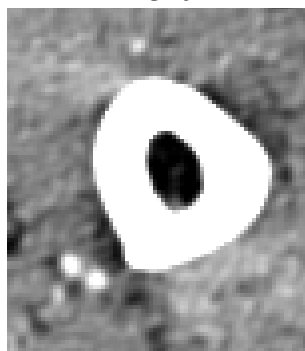

$\mathrm{Z}=255$

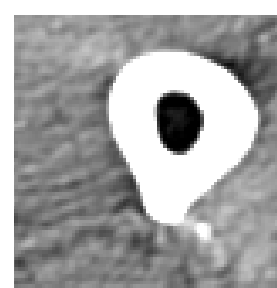

$\mathrm{z}=333$

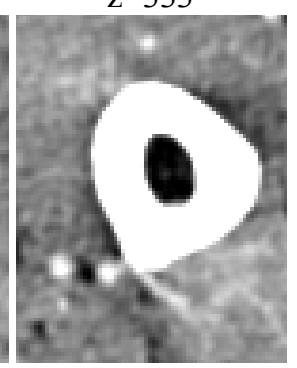

$\mathrm{z}=260$
Fig. 3. Different slices showing the different contact areas between the femur bone and the surrounding veins

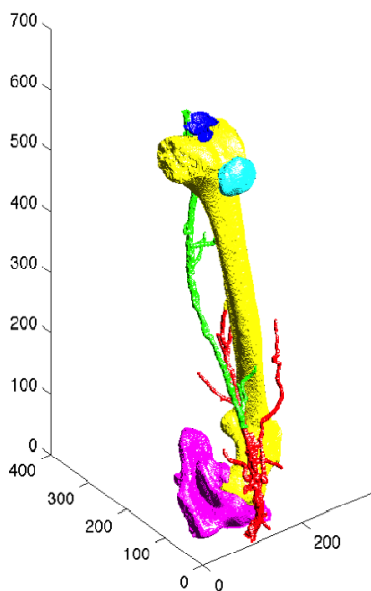

(a)

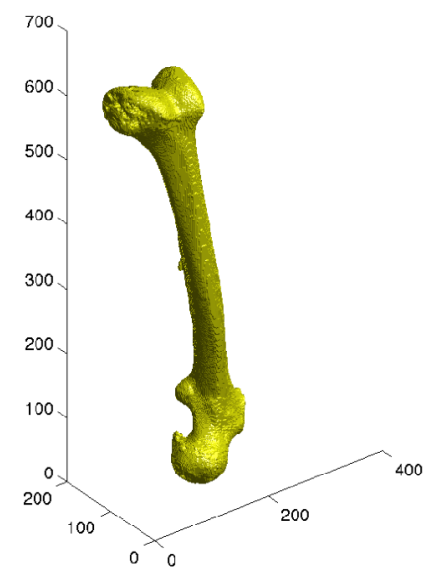

(b)
Fig. 4. A render of the final segmented image shown in Fig. 2 and Fig. 3 (a)Render showing the largest 6 objects, (b)The largest object labeled hence the femur bone.
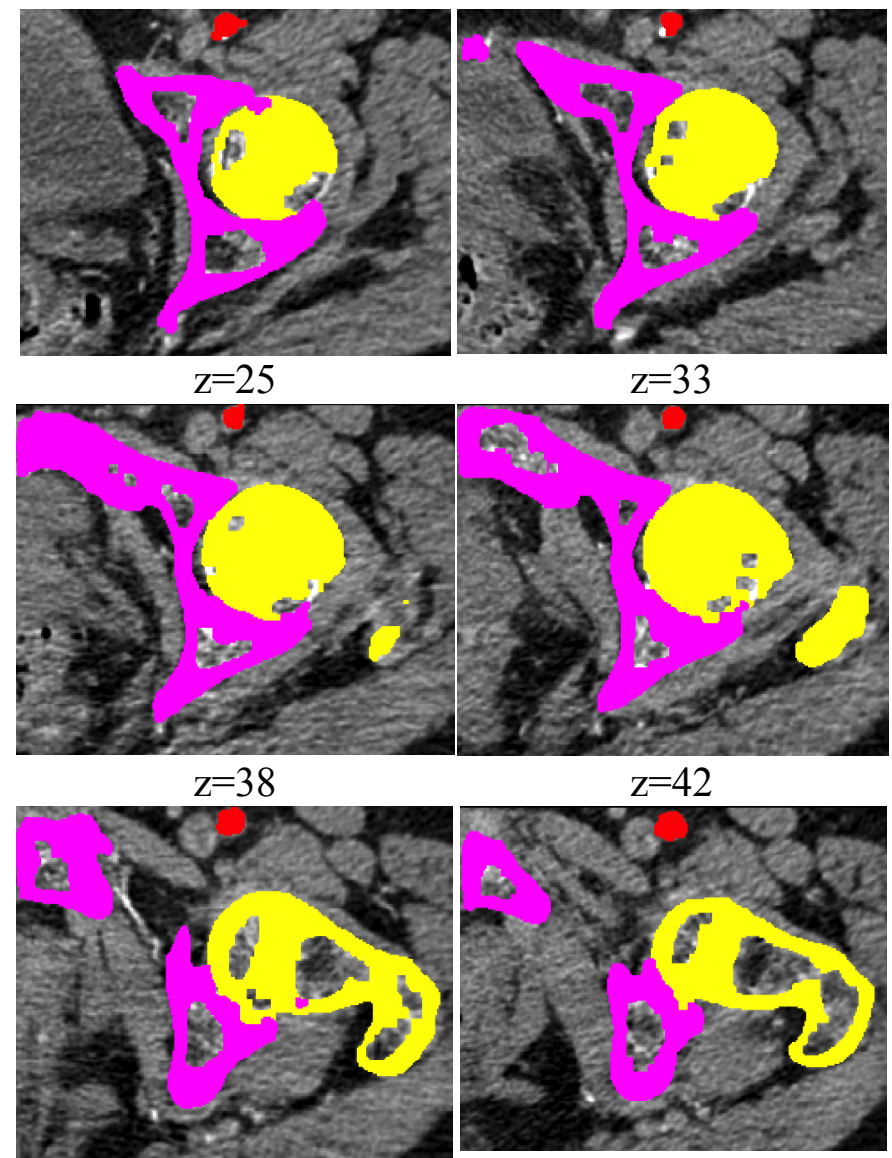

$\mathrm{Z}=53$

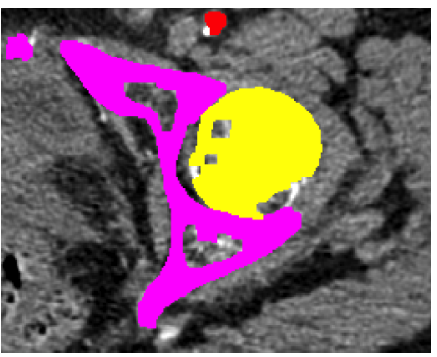

$\mathrm{z}=33$

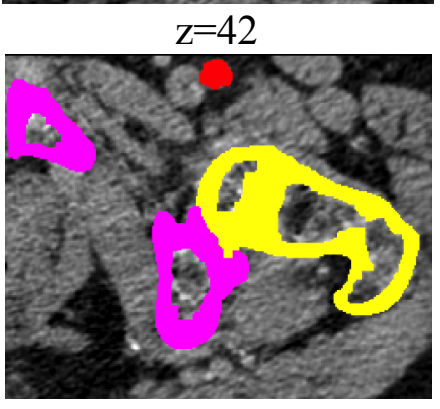

$\mathrm{Z}=58$
Fig. 5. Different slices though the hip showing the contact area between the femoral head and the acetabulum 
feature extraction process. To illustrate the problem, several slices of the connected areas between the acetabulum and the femoral head of one volume are shown Fig 2. In these images, the connections appear in different places, so the technique requires information from local features. Another problem also arises, in that the multiple contact areas between the veins in the surrounding soft tissue touching the femur, especially when a contrast agent is present. However, veins have little impact on the total surface area, as in Fig 3.

A rendering of the final labeled objects, with each label identified by a unique color, is shown in Fig. 4a. Once the femur bone is labelled and identified it can be extracted by choosing the desired label as in Fig. 4b. Successive slices through the segmented data are shown in Fig. 5. These show that the acetabulum is correctly identified as separate from the femur even when they are in contact and that the perimeters of the bones (the surfaces) are extracted correctly.
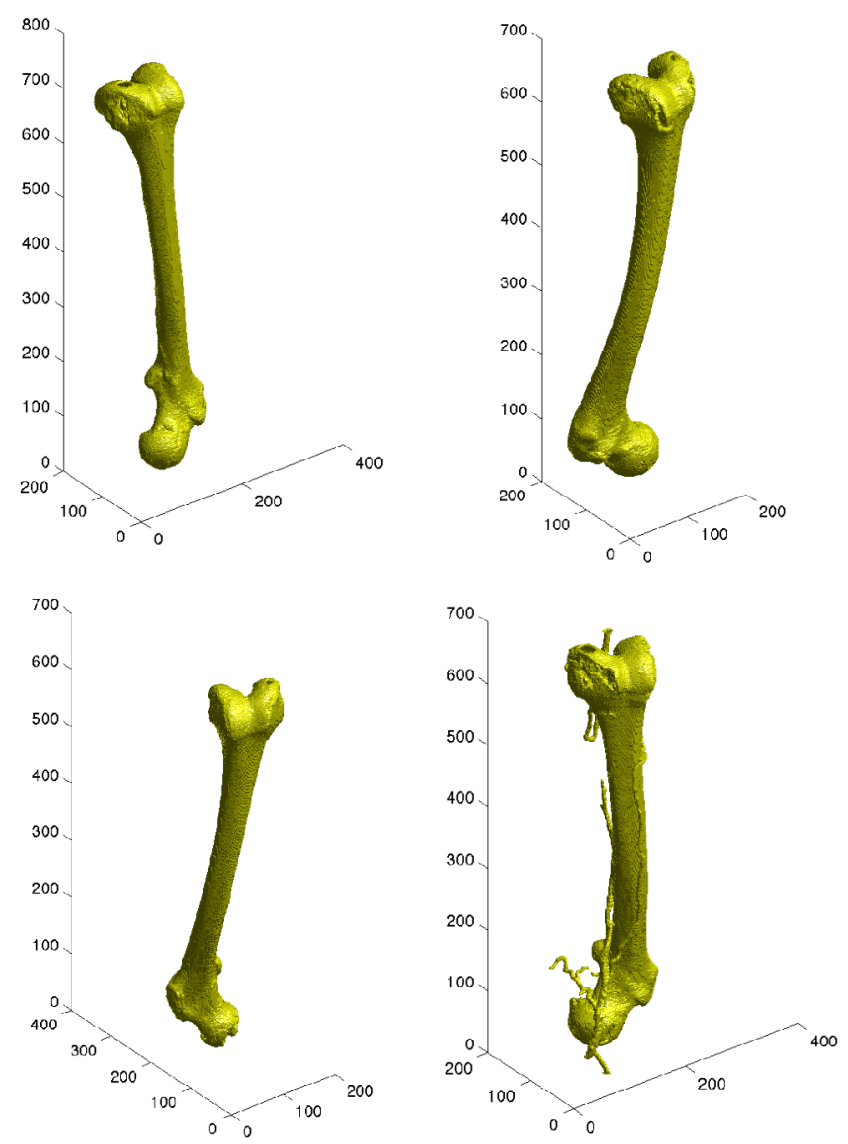

Fig. 6. Sample of four segmented femur bones using the proposed method

The proposed method has been applied to the 51 femur volumes; random sample of the results is shown in Fig. 6. The segmentation proved to be successful and the femur bone was extracted properly. Nevertheless, some of the results showed less than perfect segmentation as in Fig. 7. In some cases, some segments of the femur head were joined with the hip, in other cases the whole head of the femur bone is lost and the worst was the difficulty of separating the hip and the femur bone. A histogram provided in Fig. 8 showing the performance of the proposed method on the 51 femur bones, the effects of veins have been omitted due to their minor effect on the total surface of the segmented femur bone. This shows that a clear majority of the femurs have been successfully extracted intact, that many of the remainder have small segments missing, and that only one is extracted with connections remaining.

Due to the absence of the source code for the state of the art methods such as [6] and [7] a fair comparison would be difficult specially with variety of parameter choices available. The Dice coefficient of each sample of 9 volumetric images has been calculated. To verify the amount of loss in the total surface area in the results, the manual segmentation were used as the ground truth Fig. 9. An average of $d c=0.907$ has been calculated which indicate a minor loss in the total surface area and a successful segmentation.

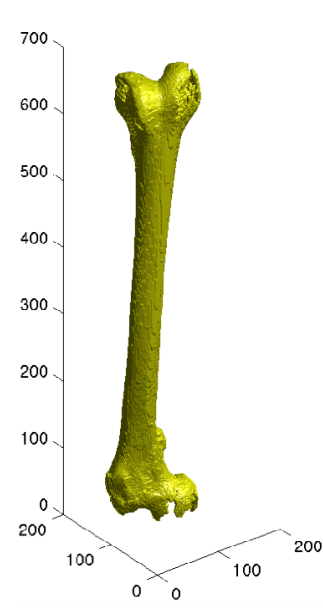

(a)

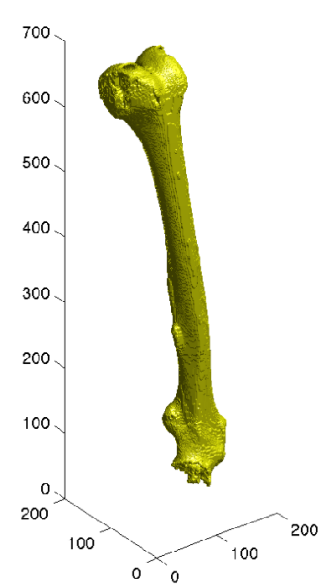

(c)

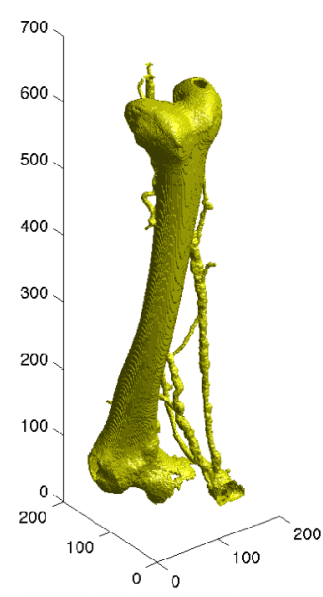

(b)

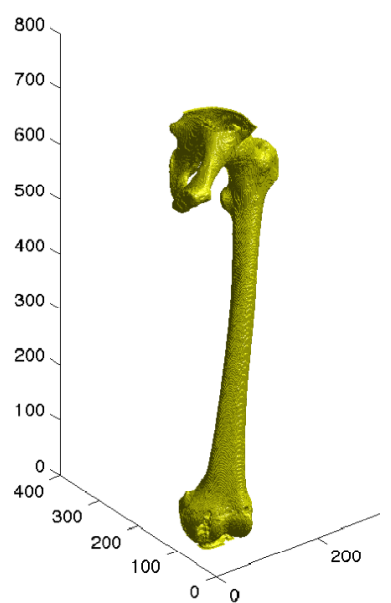

(d)
Fig. 7. Example of several situations where the proposed method did not perform well, (a) and (b) Some segments missing from the femoral head, (c) Femoral head completely lost, (d) Complete failure to separate the femur bone from the hip. 


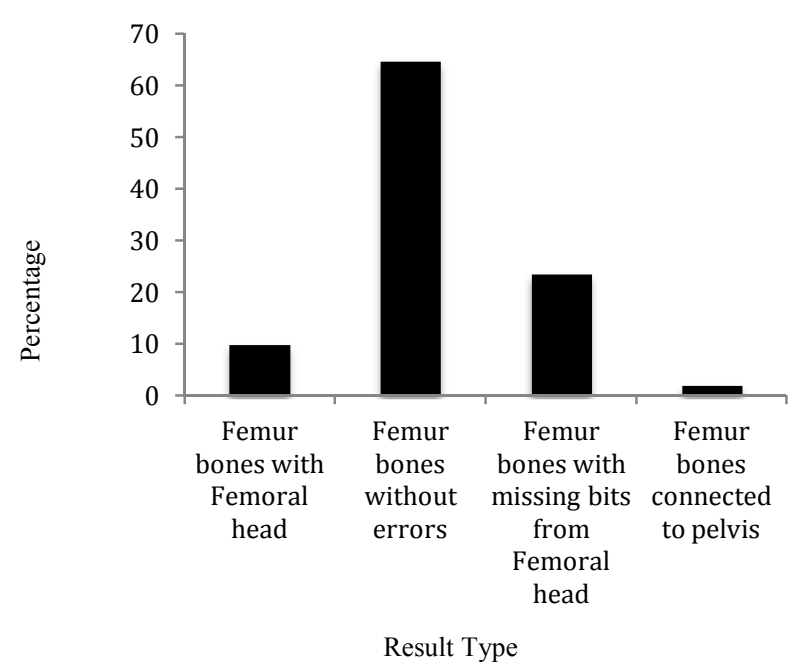

Fig. 8. A histogram showing the performance of the proposed method

Dice Coefficient per Sample

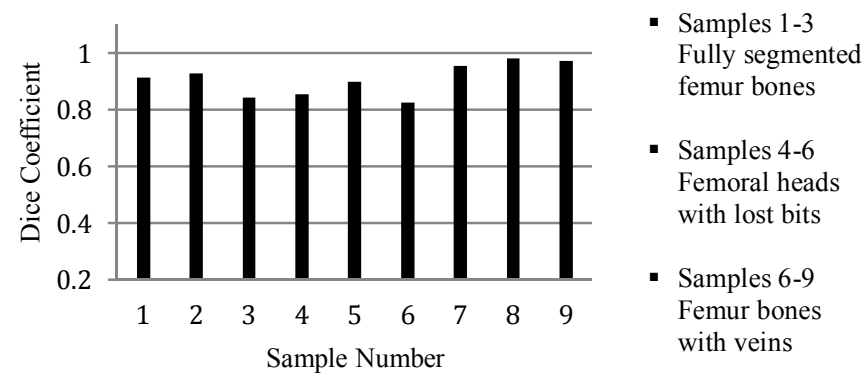

Fig. 9. Dice coefficient for a sample of the results based on the total surface area compared to the surface area of the manually segmented data.

It is also worth noting that the Dice coefficients of the femur bone extracted together with veins and arteries have introduced false-positive information. This arises from the larger surface area of the combination of bone with blood vessels and could lead the Dice coefficient to exceed unity which has not occurred here (some segments have been omitted, but cannot be observed in these renderings).

As anticipated, the proposed method showed promising results in segmenting and visualizing other bones such as the tibia and fibula bones in Fig. 10 where the only variable changed is the threshold used for the pressure (Eq. 3). Figure 11 shows images of the complete pelvis, Fig. 11a includes some blood vessels, two femoral heads and the lower lumbar region. Fig. $11 \mathrm{~b}$ shows the labelled pelvis extracted alone from this data. Again, the only variable changed is the pressure threshold and the remainder of the technique is wholly automatic.

\section{Discussion AND CONCLUSIONS}

Segmenting and visualizing the femur bone is the first step for future preclinical analysis in arthritis. The literature flourishes with machine learning techniques that depends on manually segmented data. The problem with those techniques is the general problem of machine learning, they are data oriented. Morphology operations alone would introduce or neglect some of the valuable information by acting in an isotropic manner, which would result in undesirable results. On the other hand, the new pressure analogy depends on local image features; it is anisotropic, non-iterative. The technique could even provide a mask without the need for training or learning. The proposed method based on the pressure analogy carries of the same advantages, and can handle similar density connection between the objects. This would benefit the automation of the other techniques discussed in literature such as providing an automatic large set of data for machine learning and presenting the general Hough transform with a standard template. The results can also be of great help to the preclinical studies of hip replacement, and help determining the optimum positioning of the femur bone implant to achieve a perfect fit. The proposed method has shown encouraging results for separating touching objects with the same density in computer tomography volumes. In future, more local features will be taken into account to reveal more information about the object of interest.

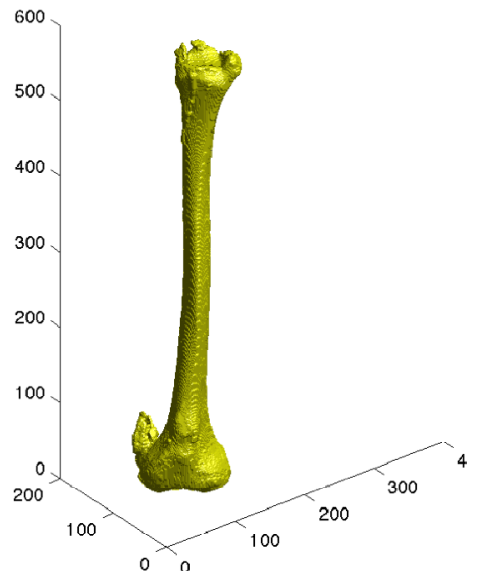

(a)

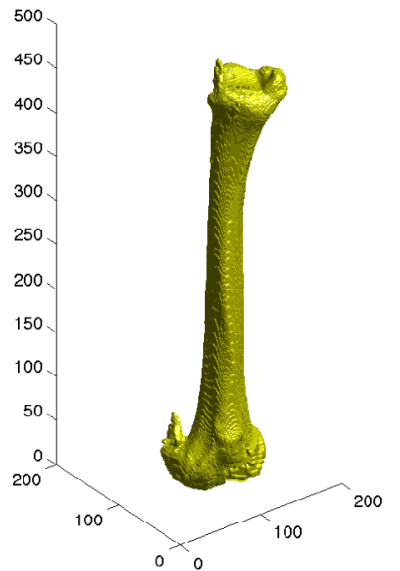

(c)

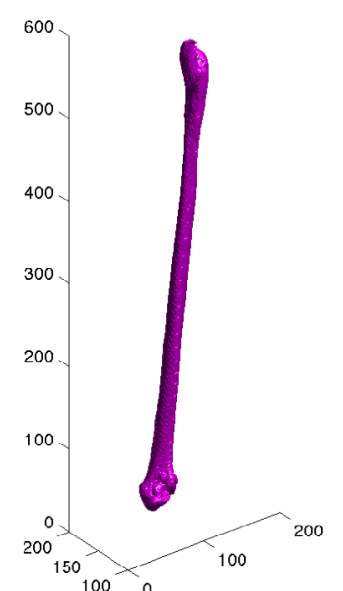

(b)

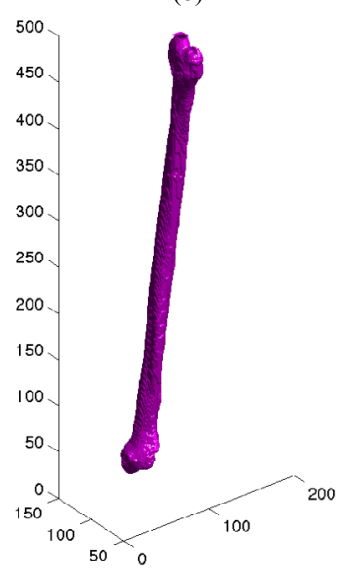

(d)
Fig. 10. Other bones segmented using the proposed method, (a) and (c) Rendering of segmented tibia bone, (b) and (d) Rendering of segmented fibula bone. 


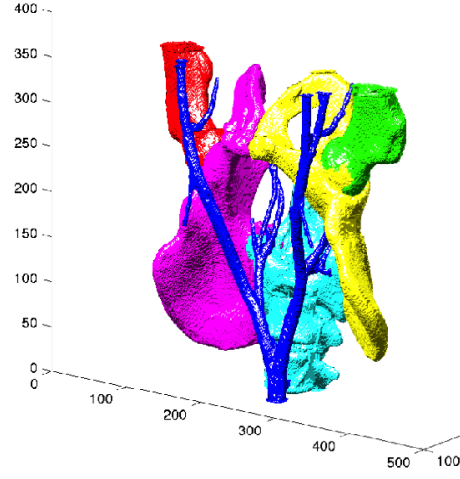

(a)

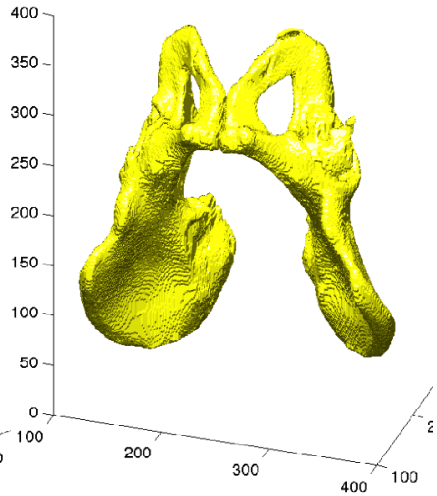

(b)
M. Krcah, G. Szekely, and R. Blanc, "Fully Automatic and Fast Segmentation of The Femur Bone from 3D-CT Images with No Shape Prior," 2011 IEEE Int. Symp. Biomed. Imaging From Nano to Macro, pp. 2087-2090, 2011.

[7] J. Zhang, C.-H. Yan, C.-K. Chui, and S.-H. Ong, "Fast Segmentation of Bone in CT Images Using 3D Adaptive Thresholding," Comput. Biol. Med., vol. 40, no. 2, pp. 231-6, Feb. 2010 .

[8] T. S. Alathari and M. S. Nixon, "Segmenting Objects in 3d Images by Volumetric Analysis," Proc. ISVC 2013, LNCS, 8033, pp. 238 $245,2013$.

Fig. 11. Other bones segmented using the proposed method (a) Rendering of the hip area including some blood vessels, two femoral heads and the lower lumbar region, (b) Rendering of the final segmented pelvis.

\section{ACKNOWLEDGMENTS}

The authors would like to thank the Public Authority for Applied Education and Training in Kuwait for providing the financial support and studentship of T Alathari.

\section{REFERENCES}

[1] J. Schmid, J. Kim, and N. Magnenat-Thalmann, "Robust Statistical Shape Models for MRI Bone Segmentation in Presence of Small Field of View," Med. Image Anal., vol. 15, no. 1, pp. 155-68, Feb. 2011.

[2] F. Yokota, T. Okada, M. Takao, N. Sugano, Y. Tada, and Y. Sato, "Automated Segmentation of The Femur and Pelvis from 3D CT Data of Diseased Hip Using Hierarchical Statistical Shape Model of Joint Structure," Med. Image Comput. Comput. Assist. Interv., vol. 12, no. Pt 2, pp. 811-8, Jan. 2009

[3] H. Seim, D. Kainmueller, and M. Heller, "Automatic Segmentation of the Pelvic Bones from CT Data Based on a Statistical Shape Model," Eurographics Workshop Vis. Comput. Biomed., pp. 93100, 2008.

[4] J. Pettersson, H. Knutsson, and M. Borga, “Automatic Hip Bone Segmentation Using Non-Rigid Registration," 18th Int. Conf. Pattern Recognit., pp. 946-949, 2006.

[5] R. a Zoroofi, Y. Sato, T. Sasama, T. Nishii, N. Sugano, K. Yonenobu, H. Yoshikawa, T. Ochi, and S. Tamura, "Automated Segmentation of Acetabulum and Femoral Head from 3-D CT Images," IEEE Trans. Inf. Technol. Biomed., vol. 7, no. 4, pp. 329 43, Dec. 2003.
L. Lam, S.-W. Lee, and C. Y. Suen, "Thinning Methodologies-A Comprehensive Survey," IEEE Trans. Pattern Anal. Mach. Intell., vol. 14 , no. 9, p. 879, 1992.

[10] T. Sørensen, “A method of Establishing Groups of Equal Amplitude in Plant Sociology Based on Similarity of Species and its Application to Analyses of the Vegetation on Danish Commons," Biol. Skr., vol. 5, pp. 1-34, 1948. 